\title{
Evaluation of Bread Wheat (Triticum aestivum L.) Breeding Lines for Yield and Yield Related Characters in Horo Guduru Wollega Zone, Western Ethiopia
}

\author{
Negash Geleta*, Desalegn Negasa and Dereje Teshome
}

\author{
College of Agriculture and Natural Resources, Wollega University, P.O Box: 395, Nekemte, Ethiopia
}

\begin{tabular}{|c|c|}
\hline Abstract & Article Information \\
\hline \multirow{15}{*}{$\begin{array}{l}\text { A total of thirty-six bread wheat (Triticum aestivum L.) breeding lines were evaluated for yield } \\
\text { and yield related traits at high and midland environments of Horo Guduru Wollega Zone, western } \\
\text { Ethiopia during } 2013 \text { cropping season. The genotypes were grown in a } 6 \text { x } 6 \text { simple lattice } \\
\text { design. Data were collected for } 12 \text { morpho-agronomic characters. Since heterogeneity of } \\
\text { variance test due to location was significant for most of traits, separate analyses for ANOVA, } \\
\text { correlation and principal components were done. The differences between two means for } \\
\text { significances were tested using SNK. The results of ANOVA showed that significant differences } \\
\text { were observed among the breeding lines at both locations for DH, PH, SPL, SPS, BY, GY and } \\
\text { TKW; while for DM and GFP at Gitilo and for HI at Guduru. At both locations, genotypes which } \\
\text { showed superior performances compared to the standard checks 'Danada' and 'Kubsa' for } \\
\text { biomass yield, grain yield, thousand kernel weight and harvest index include: ETBW7238, } \\
\text { ETBW7235, ETBW7220, ETBW7191, ETBW7199, ETBW7182, ETBW7204, ETBW7258, } \\
\text { ETBW7264, ETBW7247; and these genotypes are selected for multi-location trials for wide } \\
\text { adaptations. Principal components analysis showed that the first four PCs contributed about } \\
81.06 \% \text { of the total phenotypic variation in the genotypes at Gitilo; while the first three PCs } \\
\text { contributed about } 77.01 \% \text { to the total phenotypic variations in the genotypes at Guduru. } \\
\text { Analysis for traits association showed that at Gitilo, GY had strong and positive associations with } \\
\text { SPL and BY, indicating that genotypes with long spike had more kernels or yield than the short } \\
\text { spike types. Similarly, at Guduru, GY was significantly and positively correlated with SPS, BY } \\
\text { and HI. At both locations, traits which showed strong associations with GY could be used for } \\
\text { indirect selection criteria during genotypes evaluation for better adaptabilities to the environment. } \\
\text { Copyright@2015 STAR Journal, Wollega University. All Rights Reserved. }\end{array}$} & Article History: \\
\hline & Received : 12-01-2015 \\
\hline & Revised : 13-03-2015 \\
\hline & Accepted : 16-03-2015 \\
\hline & Keywords: \\
\hline & Breeding lines \\
\hline & Correlations \\
\hline & Ethiopia \\
\hline & Horo Guduru \\
\hline & rincipal component \\
\hline & \\
\hline & ${ }^{*}$ Corresponding Author: \\
\hline & Negash Geleta \\
\hline & \\
\hline & ayananegash@yahoo.com \\
\hline
\end{tabular}

\section{INTRODUCTION}

Wheat is the major cereal food crop in the world and also in Ethiopia. Two types of wheat are majorly produced in Ethiopia: durum wheat (tetraploid wheat, $2 \mathrm{n}=4 \mathrm{x}=28 ; T$. durum Desif.) and bread wheat (hexaploid wheat, $2 \mathrm{n}=4 \mathrm{x}=28$; T. aestivum L.) (Geleta and Grausgruber, 2011; Geleta and Grausgruber, 2013a, b). However, emmer wheat (Triticum dicoccon Schrank) is produced in marginal lands in Ethiopia (Zaharieva et al., 2010). Bread wheat (Triticum aestivum L.) has originated from natural hybrids of three diploid wild progenitors native to the Middle East. These are T. monococcum, T. tauschii (syn. Aegilops squarosa) and Aegilops speltoides (Lelley, 1976; Ribaut et al., 2001). It is an annual cool season cereal crop but it can grow in a wide range of environments around the world. Its production is highly concentrated between the latitudes of $30^{\circ}$ and $60^{\circ} \mathrm{N}$, and $27^{\circ}$ to $40^{\circ} \mathrm{S}$ (Heyne, 1987), and within the temperature range of 3 to $32{ }^{\circ} \mathrm{C}$. Wheat can grow best on well-drained soil from sea level to about 3000 m.a.s.l.

About one third of the developing world's wheat area is located in environments that are regarded as marginal for wheat production because of drought, heat and soil problems. Gains in wheat productivity in marginal environments are important because it is unlikely that increased productivity in the favourable environments will be sufficient to meet the projected growth in demand for wheat from the present to 2020 . The demand for wheat is projected to be 40 percent greater than its current level of 552 million metric tonnes by 2020 (Rosegrant, 1997: Cited in Lantican et al., 2002). Environmental stress is the most important factor, which affects crop production. According to Christiansen and Lewis (1982) only about $10 \%$ of world arable land may be classified into non-stress category. About $20 \%$ of the land is limited by mineral stress, $26 \%$ by drought stress and $15 \%$ by freezing stress. Modifying the environment for proper crop growth means the alleviation of environmental stresses through the current crop management practices (Arkin and Taylor, 1983). In many cases it may not be possible to modify the environment but the genetic modification of plants to successfully grow in stress conditions is feasible or at least has been demonstrated to be feasible.

Of the total arable land of Ethiopia, more than $50 \%$ is classified as semi-arid and arid agro ecological zones. Moisture stress is the major problem in such areas. In semi-arid and arid areas, rainfall is inadequate, erratic and 
Negash Gelata et al.,

non-uniform in distribution. Moreover, because of degradation and poor vegetation cover, soils in semi-arid and arid area have low fertility with poor water holding capacity. In addition to the above-mentioned problems, weeds also compete with the food crops for the meagre available moisture (Reddy and Kidane, 1991); besides, there are occasional out breaks of pests and diseases. In Ethiopia, wheat is an introduced crop, although its time of introduction is immemorial (Hailu, 1991), and this crop is one of Ethiopian's staple foods and the main source of calorie in the major producing regions; and it is grown between latitudes of $6^{0}$ to $16^{\circ} \mathrm{N}$, and $35^{\circ}$ to $42^{\circ} \mathrm{E}$, with altitudes ranging from 1500 to 2800 m.a.s.l. Wheat stands fourth and $3^{\text {rd }}$ in total national area and grain production respectively; and $2^{\text {nd }}$ in yield per hectare (CSA, 2014). Grain yield and yield related traits are highly variable and significantly modified by environmental factors. The effectiveness of selection depends on the amount of variability present in the genetic material for grain yield and related traits. The relative influence of various traits and their degree of associations can be estimated statistically by correlation (Dewey and Lu, 1959). Determination of relationships of characters can help to identify traits of economic importance. Hybridization provides a chance to combine the desirable characters from two or more lines into a single genotype. The effectiveness, however, depends on the genetic divergence among the lines being crossed. The greater the divergence, the greater are the chances of developing superior yielding genotypes. The present study was carried out with the objectives to select the promising variety/ies for yield and other desirable agronomic traits and to estimate the magnitude of correlation among grain yield and other agronomic traits.

\section{MATERIALS AND METHODS}

\section{Descriptions of the Study Areas}

The experiment was carried out at two districts (i.e. Gitilo experimental site in Horo woreda and Guduru Animal Production and Research Centre, in Guduru Woreda) of Horo Guduru Wollega Zone, Western Ethiopia. Gitilo experimental site and Guduru Animal Production and Research Centre, represents the highland and mid altitude environments of the wheat growing areas in the Zone respectively. Gitilo is located at 07012 ' N and $39020^{\prime} \mathrm{W}$, and altitude 2800 masl; it receives mean minimum and maximum temperature of 9 and $23{ }^{\circ} \mathrm{C}$ respectively; and it receives annual precipitation of 1844 $\mathrm{mm}$. Guduru is located at $080^{\circ} 04^{\prime} \mathrm{N}$ and $390^{\circ} 00^{\prime} \mathrm{W}$ and altitude 1760 masl; it has annual mean minimum and maximum temperature of 16 and $26{ }^{\circ} \mathrm{C}$ respectively, and it receives annual rainfall of $1817 \mathrm{~mm}$.

\section{Experimental Materials}

Thirty-six bread wheat breeding lines including two standard checks varieties were selected from 121 materials (bread wheat lines) grown in preliminary yield trial at Shambu during the 2012 cropping season. The 121 genotypes were obtained from Kulumsa Agricultural Research Centre, National Wheat Research Coordination Centre.

\section{Experimental Design and Trial Management}

The trials were established in the field on July 17 , 2013 at Gitilo and on July 19, 2013 at Guduru. The genotypes were grown in $6 \times 6$ simple lattice design. Each plot had five rows of $2.5 \mathrm{~m}$ length with $20 \mathrm{~cm}$ spacing between rows. A $1.5 \mathrm{~m}$ space was left between blocks.
Sci. Technol. Arts Res. J., Jan-March 2015, 4(1): 01-08

Recommended rates of the non-experimental variables for the specific testing site such as seed and fertilizer were used. Hence, 100/100 Urea/DAP kg/ha was used for Guduru and 100/90 Urea/DAP kg/ha was used for Gitilo. A seed rate of $150 \mathrm{~kg} / \mathrm{ha}$ was used at both locations. All other pre and post-stand establishment management practices were done as required.

\section{Data Collection}

Data were collected for the following attributes: Days to $50 \%$ heading (DH); days to $95 \%$ maturity (DM); Grain filling period (GFP); grain yield (GY); thousand seed weight (TSW); biological yield (BY); harvest index $(\mathrm{HI})$, tillers/plant (TPP); plant height (PH); number of grains per spike (NGS); spikelet per spike (SPS) and spike length (SPL).

\section{Statistical Data Analysis}

All statistical data analyses procedures were done following the procedures of Gomez and Gomez (1984) and computed using SAS software, version 9.0, 2002, USA. Since test for hmogeniety of variance for locations showed significant for most of the traits, separate analysis of variance and other subsequent tests were done for each location. The significance between two means were tested using SNK method at $5 \%$ probability level. In addition principal component analysis was carried out to identify traits which contributed more for genotypes differentiation. Pearson's simple phenotypic correlation coefficients between two pairs of traits were calculated from the phenotypic and environmental co-variances obtained by covariance analysis for the traits showed significant differences in ANOVA.

\section{RESULTS}

\section{Analysis of Variance}

At highland (Gitilo) site, the results of analysis of variance showed that there were significant differences $(P \leq 0.01)$ among the genotypes for all traits except for tiller number per plant, number of grains per spike and harvest index (Table 1). The coefficient of variation and range values was higher for GY, BY and TKW indicating that the genotypes were variable. Similarly, at Guduru significant differences $(P \leq 0.01)$ were observed among the genotypes for traits including $\mathrm{DH}, \mathrm{PH}, \mathrm{SPL}, \mathrm{SPS}, \mathrm{BY}, \mathrm{GY}$ and TSW and for $\mathrm{HI}$ at $P \leq 0.05$ (Table 2). The coefficients of variation and range values for GFP, BY, GY and $\mathrm{HI}$ were higher for the genotypes indicating further variability to select genotypes of desirable traits. The characters which showed significant differences among the genotypes at each location are used for the subsequent statistical analyses like correlation and principal component analysis. Further, these traits are important for designing future breeding scheme in the genotypes.

\section{Mean and Range Values for Phenologic and Vegetative Parameters \\ At Gitilo, the mean values for DH, DM, GFP, PH and} TPP and SPL were presented in Table 3. Mean values of the genotypes for DH, DM, GFP, PH, TPP, and SPL ranged from 71 days to 91.0 days, 125 days to 141 days, 41 to 60 days, 67 to $95 \mathrm{~cm}, 2.0$ to 3.40 , and 6.0 to 11.0 $\mathrm{cm}$, respectively. The proportion of the genotypes which gave above the average mean of the genotypes for $\mathrm{DH}$, DM, GFP, PH, TPP, and SPL were 47.22, 36.11, 55.56, $47.22,47.22$, and $36.11 \%$, respectively. The average mean values of the genotypes for the above traits were 80.80 days, 131.74 days, 50.31 days, $81.16 \mathrm{~cm}, 2.88$, and 
Table 1: Mean square values for different agronomic traits in bread wheat genotypes tested at Gitilo, 2013

\begin{tabular}{llllll}
\hline \multicolumn{1}{c}{ Traits } & MSB (Df=1) & MSG(Df=35) & MSE(Df=35) & \multicolumn{1}{c}{ Min. } & Max. \\
\hline Days to 50\% heading & 24.50 & $53.64^{\star * *}$ & 2.50 & 70.00 & 93.00 \\
Days to 90 \% maturity & 0.014 & $18.53^{\star * *}$ & 3.014 & 124.00 & 141.00 \\
Grain filling period & 9.39 & $52.67^{\star * *}$ & 7.39 & 39.00 & 61.00 \\
Plant height, cm & 0.117 & $67.28^{* * *}$ & 1.30 & 66.50 & 95.30 \\
Tiller number per plant & 0.061 & $0.25 \mathrm{~ns}$ & 0.15 & 1.50 & 3.50 \\
Spike length, cm & 0.014 & $4.78^{\star * *}$ & 0.014 & 6.00 & 11.00 \\
Spikelets per spike & 2.00 & $5.30^{\star * *}$ & 1.37 & 12.00 & 20.00 \\
Number of grains per spike & 1.84 & $0.185 \mathrm{~ns}$ & 0.15 & 2.20 & 4.50 \\
Biological yield (t/ha) & 23.99 & $9.88^{\star * *}$ & 2.106 & 6.01 & 18.44 \\
Grain yield (t/ha) & 7.17 & $3.27^{* * *}$ & 0.59 & 2.48 & 8.62 \\
Harvest index & 0.0012 & $0.0025 \mathrm{~ns}$ & 0.003 & 0.30 & 0.60 \\
Thousand grain weight, g & 0.014 & $22.67^{\star * *}$ & 1.014 & 33.00 & 49.00 \\
\hline
\end{tabular}

${ }^{*}$ and ${ }^{* *}$ significant at $\mathrm{P} \leq 0.05$ and $\mathrm{P} \leq 0.01$, respectively; Df- degrees of freedom.

Table 2: Mean square values for different agronomic traits in bread wheat genotypes tested at Guduru, 2013

\begin{tabular}{lllccc}
\hline Traits & MSB (Df=1) & MSG (Df=35) & MSE (Df=35) & Min. & Max. \\
\hline Days to 50\% heading & 8.68 & $46.19^{* * *}$ & 9.51 & 53.00 & 75.00 \\
Days to 90\% maturity & 5.01 & $113.10 \mathrm{~ns}$ & 64.93 & 91.00 & 126.00 \\
Grain filling period & 26.89 & $65.51 \mathrm{~ns}$ & 52.80 & 21.00 & 68.00 \\
Plant height, cm & 194.08 & $65.98^{\star *}$ & 26.80 & 31.55 & 76.30 \\
Tiller number per plant & 0.67 & $0.51 \mathrm{~ns}$ & 0.30 & 1.44 & 5.100 \\
Spike length, cm & 1.46 & $1.51^{* * *}$ & 0.42 & 3.65 & 9.100 \\
Spikelet per spike & 5.02 & $4.56^{* * *}$ & 1.66 & 6.44 & 14.22 \\
Number of grains per spike & 0.002 & $0.221 \mathrm{~ns}$ & 0.28 & 2.00 & 4.50 \\
Biological yield (t/ha) & 0.73 & $3.78^{\star * *}$ & 1.20 & 2.36 & 8.83 \\
Grain yield (t/ha) & 0.158 & $0.85^{* * *}$ & 0.21 & 0.66 & 3.62 \\
Harvest index & 0.0001 & $0.016^{*}$ & 0.008 & 0.15 & 0.80 \\
Thousand grain weight, g & 8.000 & $19.26^{* *}$ & 8.31 & 28.00 & 45.00 \\
\hline
\end{tabular}

${ }^{*}$ and ${ }^{* *}$ significant at $\mathrm{P} \leq 0.05$ and $\mathrm{P} \leq 0.01$, respectively; Df- degrees of freedom; ns- non-significant.

Table 3: Mean values for different phenology and vegetative traits in bread wheat genotypes tested at Gitilo, 2013

\begin{tabular}{|c|c|c|c|c|c|c|c|}
\hline S/No & Genotypes & DH & DM & GFP & $\mathrm{PH}(\mathrm{cm})$ & TPP & SPL \\
\hline 1 & ETBW 7178 & 84.5 cdef & $135.5 \mathrm{bcd}$ & 51.0 abcdefg & 84.50 bcde & 2.85 & $10.0 \mathrm{c}$ \\
\hline 2 & ETBW 7252 & $86.0 \mathrm{cde}$ & 133.0 bcde & 47.0 bcdefg & 81.40 defghijk & 2.45 & $10.5 b$ \\
\hline 3 & ETBW 7238 & $93.0 \mathrm{a}$ & $140.5 \mathrm{a}$ & $42.5 \mathrm{fg}$ & 79.05 hijkl & 3.25 & $11.0 \mathrm{a}$ \\
\hline 4 & ETBW 7198 & 84.5 cdef & $132.5 \mathrm{bcde}$ & 47.0 bcdefg & $71.40 n$ & 3.40 & $8.0 \mathrm{e}$ \\
\hline 5 & Kubsa & $76.0 \mathrm{jkl}$ & 130.5 cdef & $54.5 \mathrm{abcd}$ & $95.25 a$ & 3.20 & $9.0 \mathrm{~d}$ \\
\hline 6 & ETBW 7237 & $91.0 \mathrm{ab}$ & 133.0 bcde & $41.0 \mathrm{~g}$ & $76.30 \mathrm{Im}$ & 2.65 & $9.0 \mathrm{~d}$ \\
\hline 7 & ETBW 7171 & $83.0 \mathrm{cdefgh}$ & $131.5 \mathrm{bcde}$ & 48.5 bcdefg & 80.75 efghijk & 3.15 & $8.0 \mathrm{e}$ \\
\hline 8 & ETBW 7208 & $87.5 \mathrm{bcd}$ & 133.0 bcde & 45.5 bcdefg & $94.25 \mathrm{a}$ & 3.15 & $9.0 \mathrm{~d}$ \\
\hline 9 & ETBW 7236 & $84.5 \mathrm{cdef}$ & 128.0 ef & $43.5 \mathrm{efg}$ & 67.30 o & 3.15 & $7.0 \mathrm{f}$ \\
\hline 10 & ETBW 7248 & 81.5 efghij & 129.0 def & 47.5 bcdefg & $70.35 n$ & 2.50 & $6.0 \mathrm{~g}$ \\
\hline 11 & ETBW 7173 & $85.5 \mathrm{cde}$ & 129.5 def & 44.0 defg & 77.75 jkl & 3.20 & $8.0 \mathrm{e}$ \\
\hline 12 & ETBW 7235 & $88.5 \mathrm{BC}$ & 129.5 def & $41.0 \mathrm{~g}$ & 81.25 defghijk & 2.70 & $7.0 \mathrm{f}$ \\
\hline 13 & ETBW 7268 & 84.5 cdef & 127.5 ef & $43.0 \mathrm{fg}$ & 84.25 bcdef & 3.20 & $6.0 \mathrm{~g}$ \\
\hline 14 & ETBW 7174 & $75.5 \mathrm{kl}$ & 131.5 bcdef & $56.0 \mathrm{ab}$ & $87.45 \mathrm{~b}$ & 2.90 & $8.0 \mathrm{e}$ \\
\hline 15 & ETBW 7220 & $77.0 \mathrm{ijkl}$ & $132.0 \mathrm{bcde}$ & $55.0 \mathrm{abc}$ & 85.35 bcd & 2.60 & $7.0 \mathrm{f}$ \\
\hline 16 & ETBW 7221 & 77.5 ghijkl & $132.0 \mathrm{bcde}$ & $54.5 \mathrm{abcd}$ & 84.05 bcdef & 2.35 & $6.0 \mathrm{~g}$ \\
\hline 17 & ETBW 7227 & $75.5 \mathrm{kl}$ & 132.5 bcde & $59.0 \mathrm{a}$ & 35 bc & 3.15 & $8.0 \mathrm{e}$ \\
\hline 18 & ETBW 7239 & $75.5 \mathrm{kl}$ & 128.0 ef & 52.5 abcdef & 84.65 bcde & 3.00 & $9.0 \mathrm{~d}$ \\
\hline 19 & ETBW 7160 & $77.0 \mathrm{ijkl}$ & $128.5 \mathrm{def}$ & $51.5 \mathrm{abcdefg}$ & $85.35 \mathrm{bcd}$ & 3.25 & $9.0 \mathrm{~d}$ \\
\hline 20 & ETBW 7161 & 79.0 fghijk & 132.0 bcde & 48.5 bcdefg & 84.20 bcdef & 2.8 & $10.0 \mathrm{c}$ \\
\hline 21 & ETBW 7191 & $75.5 \mathrm{kl}$ & 128.5 def & 52.5 abcdef & 81.70 defghij & 2.85 & $10.0 \mathrm{c}$ \\
\hline 22 & ETBW 7199 & 83.5 cdefg & $131.5 \mathrm{bcdef}$ & 48.0 bcdefg & 83.30 cdefg & 3.10 & $11.0 \mathrm{a}$ \\
\hline 23 & ETBW 7182 & 78.0 ghijkl & $128.5 \mathrm{def}$ & 50.5 abcdefg & 81.00 efghijk & 2.80 & $10.0 \mathrm{c}$ \\
\hline 24 & ETBW 7194 & 78.5 ghijkl & $131.0 \mathrm{bcdef}$ & 52.5 abcdef & 80.20 fghijk & 3.15 & $6.0 \mathrm{~g}$ \\
\hline 25 & ETBW 7204 & 78.5 ghijkl & 131.0 bcdef & 52.5 abcdef & $78.05 \mathrm{jkl}$ & 3.30 & $7.0 \mathrm{f}$ \\
\hline 26 & ETBW 7234 & $73.0 \mathrm{~lm}$ & $131.5 \mathrm{bcdef}$ & $56.0 \mathrm{ab}$ & 79.55 ghijkl & 2.00 & $8.0 \mathrm{e}$ \\
\hline 27 & ETBW 7164 & 84.5 cdef & 132.5 bcde & 48.0 bcdefg & $86.40 \mathrm{bc}$ & 3.30 & $10.0 \mathrm{c}$ \\
\hline 28 & ETBW 7195 & 78.0 ghijkl & $133.5 \mathrm{bcde}$ & $55.5 \mathrm{abc}$ & 79.15 hijkl & 2.65 & $8.0 \mathrm{e}$ \\
\hline 29 & ETBW 7244 & 80.5 efghijk & 134.5 bcde & 54.0 abcde & $77.40 \mathrm{kl}$ & 2.80 & $6.0 \mathrm{~g}$ \\
\hline 30 & ETBW 7258 & $70.5 \mathrm{~m}$ & 130.5 cdef & $60.0 \mathrm{a}$ & 82.30 defghi & 3.10 & $10.0 \mathrm{c}$ \\
\hline 31 & ETBW 7264 & $73.0 \mathrm{Im}$ & $125.0 \mathrm{f}$ & 52.0 abcdef & 82.95 cdefgh & 2.75 & $9.0 \mathrm{~d}$ \\
\hline 32 & ETBW 7215 & 78.5 ghijkl & $136.5 \mathrm{abc}$ & $59.0 \mathrm{a}$ & $74.30 \mathrm{~m}$ & 2.30 & $6.0 \mathrm{~g}$ \\
\hline 33 & ETBW 7156 & 82.5 defghi & 134.5 bcde & 52.0 abcdef & $78.75 \mathrm{ijkl}$ & 3.15 & $7.0 \mathrm{f}$ \\
\hline 34 & ETBW 7247 & 81.5 efghij & 134.5 bcde & 53.0 abcdef & 81.40 defghijk & 2.35 & $7.0 \mathrm{f}$ \\
\hline 35 & DANADA'A & 83.5 cdefg & $132.0 \mathrm{bcde}$ & 47.5 bcdefg & $71.75 n$ & 2.80 & $7.0 \mathrm{f}$ \\
\hline \multirow[t]{3}{*}{36} & ETBW 7175 & 82.5 defghi & $137.5 \mathrm{ab}$ & 45.0 cdefg & 82.45 cdefghi & 2.35 & $8.0 \mathrm{e}$ \\
\hline & MEAN & 80.80 & 131.74 & 50.31 & 81.16 & 2.88 & 8.21 \\
\hline & CV (\%) & 1.96 & 13.20 & 5.40 & 1.41 & 13.42 & 1.44 \\
\hline
\end{tabular}


Negash Gelata et alo,

$8.21 \mathrm{~cm}$ respectively. The mean values of the above traits for standard checks 'Kubsa' and 'Danada' were 76.0 and 83.50 days, 130.50 and 132.0 days, 54.50 and 47.50 days, 95.25 and $71.75 \mathrm{~cm}, 3.20$ and 2.80 , and 9.0 and 7.0 $\mathrm{cm}$, respectively.

At Guduru, the mean values for DH, DM, GFP, $\mathrm{PH}$, TPP and SPL were presented in Table 4. Mean values of the genotypes for DH, DM, GFP, PH, TPP, and SPL ranged from 54.0 days to 75.0 days, 92.0 days to 124.0 days, 35.0 to 60 days, 42.99 to $71.49 \mathrm{~cm}, 2.15$ to 4.21 ,
Sci. Technol. Arts Res. J., Jan-March 2015, 4(1): 01-08

and 4.6 to $8.10 \mathrm{~cm}$, respectively. The proportions of the genotypes which gave above the average mean of the genotypes for $\mathrm{DH}, \mathrm{DM}, \mathrm{GFP}, \mathrm{PH}, \mathrm{TPP}$, and SPL were $36.11 \%, 50.0 \%, 38.89 \%, 50.0 \%, 44.44 \%$, and 52.78 $\%$, respectively. The average mean values of the genotypes for the above traits were $63.51,105.46$ days, 41.94 days, $59.43 \mathrm{~cm}, 3.10$, and $6.29 \mathrm{~cm}$ respectively. The mean values of the above traits for standard checks 'Kubsa' and 'Danada' were 68.50 and 59.0 days, 113.50 and 96.50 days, 45.0 and 37.50 days, 62.55 and 56.89 $\mathrm{cm}, 2.94$ and 2.70 , and 6.96 and $6.65 \mathrm{~cm}$, respectively.

Table 4: Mean values for phenology and vegetative traits in bread wheat genotypes tested at Guduru, 2013

\begin{tabular}{|c|c|c|c|c|c|c|c|}
\hline S/No. & Genotypes & DH & DM & GFP & PH & TPP & SPL \\
\hline 1 & ETBW 7178 & 66.0 abcde & 104.0 & 38.0 & $64.83 a b$ & 2.75 & 7.66 abc \\
\hline 2 & ETBW 7252 & 59.5 bcde & 94.0 & 34.5 & $58.55 \mathrm{abc}$ & 4.05 & $6.49 \mathrm{abcd}$ \\
\hline 3 & ETBW 7238 & 60.0 bcde & 98.0 & 38.0 & $60.72 \mathrm{abc}$ & 2.66 & $5.77 \mathrm{abcd}$ \\
\hline 4 & ETBW 7198 & $67.5 \mathrm{abcd}$ & 115.0 & 47.5 & $58.55 \mathrm{abc}$ & 3.05 & $7.93 a b$ \\
\hline 5 & Kubsa & $68.5 \mathrm{abcd}$ & 113.5 & 45.0 & $62.55 \mathrm{abc}$ & 2.94 & $6.96 \mathrm{abcd}$ \\
\hline 6 & ETBW 7237 & 63.5 abcde & 102.5 & 39.0 & $62.66 \mathrm{abc}$ & 2.49 & $6.44 \mathrm{abcd}$ \\
\hline 7 & ETBW 7171 & 59.5 bcde & 96.5 & 37.0 & $42.99 \mathrm{c}$ & 3.27 & $4.66 \mathrm{~d}$ \\
\hline 8 & ETBW 7208 & $67.0 \mathrm{abcd}$ & 112.0 & 45.0 & $66.22 \mathrm{ab}$ & 3.10 & $5.94 \mathrm{abcd}$ \\
\hline 9 & ETBW 7236 & 64.0 abcde & 124.0 & 60.0 & $60.16 \mathrm{abc}$ & 2.93 & $6.82 \mathrm{abcd}$ \\
\hline 10 & ETBW 7248 & 63.5 abcde & 102.0 & 38.5 & 45.82 bc & 3.27 & $5.10 \mathrm{~cd}$ \\
\hline 11 & ETBW 7173 & $67.0 \mathrm{abcd}$ & 114.5 & 47.5 & $60.00 a b c$ & 2.71 & $6.82 \mathrm{abcd}$ \\
\hline 12 & ETBW 7235 & $71.5 \mathrm{ab}$ & 112.5 & 41.0 & $71.49 \mathrm{a}$ & 3.05 & $6.71 \mathrm{abcd}$ \\
\hline 13 & ETBW 7268 & 65.0 abcde & 102.5 & 37.5 & $62.99 \mathrm{abc}$ & 3.60 & $7.43 \mathrm{abc}$ \\
\hline 14 & ETBW 7174 & $57.0 \mathrm{de}$ & 111.0 & 54.0 & $57.61 \mathrm{abc}$ & 2.99 & $6.38 \mathrm{abcd}$ \\
\hline 15 & ETBW 7220 & 59.5 bcde & 109.5 & 50.0 & $63.33 \mathrm{abc}$ & 3.99 & $6.38 \mathrm{abcd}$ \\
\hline 16 & ETBW 7221 & $67.5 \mathrm{abcd}$ & 110.0 & 42.5 & $59.94 \mathrm{abc}$ & 4.21 & $6.27 \mathrm{abcd}$ \\
\hline 17 & ETBW 7227 & 66.0 abcde & 104.5 & 38.5 & $56.55 \mathrm{abc}$ & 3.55 & $5.60 \mathrm{abcd}$ \\
\hline 18 & ETBW 7239 & 60.0 bcde & 97.5 & 37.5 & $56.10 \mathrm{abc}$ & 2.54 & $5.27 \mathrm{~cd}$ \\
\hline 19 & ETBW 7160 & 59.0 bcde & 105.0 & 46.0 & $59.33 \mathrm{abc}$ & 3.27 & $5.38 \mathrm{bcd}$ \\
\hline 20 & ETBW 7161 & $67.5 \mathrm{abcd}$ & 108.5 & 41.0 & $55.22 \mathrm{abc}$ & 3.66 & $5.60 \mathrm{abcd}$ \\
\hline 21 & ETBW 7191 & 63.0 bcde & 100.5 & 37.5 & $64.44 \mathrm{ab}$ & 3.33 & $6.82 \mathrm{abcd}$ \\
\hline 22 & ETBW 7199 & 60.5 bcde & 112.0 & 51.5 & $61.54 \mathrm{abc}$ & 3.83 & $6.33 \mathrm{abcd}$ \\
\hline 23 & ETBW 7182 & $75.0 \mathrm{a}$ & 110.0 & 35.0 & $66.77 \mathrm{ab}$ & 3.49 & $5.82 \mathrm{abcd}$ \\
\hline 24 & ETBW 7194 & 58.5 cde & 106.0 & 47.5 & $55.27 \mathrm{abc}$ & 3.16 & $8.10 \mathrm{a}$ \\
\hline 25 & ETBW 7204 & 65.5 abcde & 108.0 & 42.5 & $51.75 \mathrm{abc}$ & 2.94 & $6.71 \mathrm{abcd}$ \\
\hline 26 & ETBW 7234 & 59.0 bcde & 95.0 & 36.0 & $70.15 a$ & 3.10 & $5.53 \mathrm{abcd}$ \\
\hline 27 & ETBW 7164 & $54.0 \mathrm{e}$ & 92.0 & 38.0 & $56.05 \mathrm{abc}$ & 2.10 & $5.32 \mathrm{bcd}$ \\
\hline 28 & ETBW 7195 & 62.0 bcde & 100.0 & 38.0 & $61.25 \mathrm{abc}$ & 3.40 & $5.15 \mathrm{~cd}$ \\
\hline 29 & ETBW 7244 & $68.0 \mathrm{abcd}$ & 110.0 & 42.0 & $54.44 \mathrm{abc}$ & 3.40 & $5.60 \mathrm{abcd}$ \\
\hline 30 & ETBW 7258 & 63.0 bcde & 101.0 & 38.0 & $61.00 \mathrm{abc}$ & 2.65 & $6.65 \mathrm{abcd}$ \\
\hline 31 & ETBW 7264 & 64.0 abcde & 106.5 & 42.5 & $56.96 \mathrm{abc}$ & 2.50 & $6.50 \mathrm{abcd}$ \\
\hline 32 & ETBW 7215 & $71.0 \mathrm{abc}$ & 113.0 & 42.0 & $64.28 \mathrm{ab}$ & 2.15 & $6.30 \mathrm{abcd}$ \\
\hline 33 & ETBW 7156 & 58.5 cde & 96.5 & 38.0 & $60.95 \mathrm{abc}$ & 2.70 & $5.75 \mathrm{abcd}$ \\
\hline 34 & ETBW 7247 & $70.0 \mathrm{abc}$ & 116.0 & 46.0 & $57.95 \mathrm{abc}$ & 3.35 & $8.05 a$ \\
\hline 35 & DANADA'A & 59.0 bcde & 96.5 & 37.5 & $56.89 \mathrm{abc}$ & 2.70 & $6.65 \mathrm{abcd}$ \\
\hline \multirow[t]{3}{*}{36} & ETBW 7175 & $56.5 \mathrm{de}$ & 96.5 & 40.0 & $54.10 \mathrm{abc}$ & 2.75 & $5.30 \mathrm{~cd}$ \\
\hline & MEAN & 63.51 & 105.46 & 41.94 & 59.43 & 3.10 & 6.29 \\
\hline & CV (\%) & 4.86 & 7.64 & 17.32 & 8.71 & 17.59 & 10.28 \\
\hline
\end{tabular}

\section{Yield and Yield Components}

The mean values for SPS, NGPS, BY, GY, $\mathrm{HI}$ and TKW at Gitilo were presented in Table 5. Mean values of the genotypes for SPS, NGPS, BY, GY, HI and TKW ranged from 13.0 to $19.50,2.4$ to $3.8,6.59$ to $14.80 \mathrm{t} / \mathrm{ha}$, , 2.78 to $7.56 \mathrm{t} / \mathrm{ha}, 0.35$ to 0.54 , and 33.50 to $48.0 \mathrm{~g}$, respectively. The proportion of the genotypes which gave above the average mean of the genotypes for SPS, NGPS, BY, GY, HI and TKW were 63.89, 47.22, 47.22, $50.0,41.67$, and $52.78 \%$, respectively. The average mean values of the genotypes for the above traits were 19.92, 3.02, $11.19 \mathrm{t} / \mathrm{ha}, 5.08 \mathrm{t} / \mathrm{ha}, 0.45$, and $41.99 \mathrm{~g}$ respectively. The mean values of the above traits for standard checks 'Kubsa' and 'Danada' were 17.50 and $13.50,3.40$ and $2.90,8.45$ and $9.10 \mathrm{t} / \mathrm{ha}, 3.95$ and 4.55 t/ha, 0.45 and 0.45 , and 48.0 and $33.50 \mathrm{~g}$, respectively. Genotypes which are superior to the best standard check 'danada (4.55 t/ha)' for grain yield, thousand kernel weight and harvest index include: ETBW 7252, ETBW7238, ETBW7198, ETBW7208, ETBW7235, ETBW7220, ETBW7227, ETBW7161, ETBW7191, ETBW7199, ETBW7182, ETBW7204, ETBW7258, ETBW7264, ETBW7215, ETBW7247, and ETBW7175. And these genotypes are selected for further over locations testing.

The mean values for SPS, NGPS, BY, GY, HI and TKW at Guduru were presented in Table 6. Mean values 
Negash Gelata et alo,

of the genotypes for SPS, NGPS, BY, GY, HI and TKW ranged from 7.6 to $13.53,2.10$ to $3.50,3.10$ to $8.74 \mathrm{t} / \mathrm{ha}$, , 0.78 to $3.01 \mathrm{t} / \mathrm{ha}, 0.22$ to 0.64 , and 30.50 to $41.50 \mathrm{~g}$, respectively. The proportion of the genotypes which gave above the average mean of the genotypes for SPS, NGPS, BY, GY, HI and TKW were 63.89, 30.56, 50.0, $50.0,41.67$, and $50.0 \%$, respectively. The average mean values of the genotypes for the above traits were 10.81 , $2.69,5.10 \mathrm{t} / \mathrm{ha}, 1.67 \mathrm{t} / \mathrm{ha}, 0.33$, and $35.19 \mathrm{~g}$ respectively. The mean values of the above traits for standard checks 'Kubsa' and 'Danada' were 13.10 and 11.80, 2.60 and
Sci. Technol. Arts Res. J., Jan-March 2015, 4(1): 01-08

$2.40,5.63$ and $5.83 \mathrm{t} / \mathrm{ha}, 1.71$ and $1.38 \mathrm{t} / \mathrm{ha}, 0.30$ and 0.23 ; and 33.0 and $33.0 \mathrm{~g}$, respectively. Genotypes which are superior to the best standard check 'Kubsa' for grain yield (1.71 t/ha), thousand kernel weight and harvest index include: ETBW 7178, ETBW 7238, ETBW 7236, ETBW 7248, ETBW 7173, ETBW 7235, ETBW 7174, ETBW 7220, ETBW 7191, ETBW 7199, ETBW 7182, ETBW 7204, ETBW 7234, ETBW 7258, ETBW 7264 and ETBW 7247. And these genotypes are selected for further over locations testing.

Table 5: Mean values for different grain yield \& other yield components in bread wheat genotypes tested at Gitilo, 2013.

\begin{tabular}{|c|c|c|c|c|c|c|c|}
\hline S/No. & Genotypes & SPS & NGPS & BY (t ha-1) & GY $\left(\mathrm{t} \mathrm{ha}^{-1}\right)$ & $\mathrm{HI}$ & TKW (g) \\
\hline 1 & ETBW 7178 & $18.0 \mathrm{abc}$ & 2.6 & 9.10 abcde & 4.42 bcdef & 0.46 & $47.0 \mathrm{abc}$ \\
\hline 2 & ETBW 7252 & $14.0 \mathrm{~cd}$ & 3.5 & $13.86 a b$ & $6.32 \mathrm{abcd}$ & 0.45 & 45.0 abcdef \\
\hline 3 & ETBW 7238 & $19.0 a b$ & 3.8 & $14.11 \mathrm{ab}$ & $7.56 \mathrm{a}$ & 0.54 & 45.5 abcde \\
\hline 4 & ETBW 7198 & $16.5 \mathrm{abcd}$ & 2.8 & $14.75 \mathrm{a}$ & $7.31 \mathrm{ab}$ & 0.49 & 41.5 fghij \\
\hline 5 & Kubsa & $17.5 \mathrm{abcd}$ & 3.4 & 8.45 bcde & 3.95 def & 0.45 & $48.0 \mathrm{a}$ \\
\hline 6 & ETBW 7237 & $16.5 \mathrm{abcd}$ & 2.8 & 11.17 abcde & 5.12 abcdef & 0.46 & $38.5 \mathrm{jklmn}$ \\
\hline 7 & ETBW 7171 & $15.5 \mathrm{abcd}$ & 3.1 & $12.24 \mathrm{abcd}$ & 5.36 abcdef & 0.43 & $36.0 n$ \\
\hline 8 & ETBW 7208 & $16.0 \mathrm{abcd}$ & 3.2 & $14.80 \mathrm{a}$ & $7.04 \mathrm{abc}$ & 0.48 & 44.5 bcdefg \\
\hline 9 & ETBW 7236 & $16.0 \mathrm{abcd}$ & 2.6 & 10.98 abcde & 5.26 abcdef & 0.48 & 39.5 ijklm \\
\hline 10 & ETBW 7248 & $16.5 \mathrm{abcd}$ & 2.6 & $6.59 \mathrm{e}$ & $2.78 \mathrm{~F}$ & 0.42 & $47.5 \mathrm{ab}$ \\
\hline 11 & ETBW 7173 & $16.0 \mathrm{abcd}$ & 2.8 & 10.32 abcde & 4.31 bcdef & 0.41 & $38.0 \mathrm{klmn}$ \\
\hline 12 & ETBW 7235 & $17.0 \mathrm{abcd}$ & 2.8 & $12.49 \mathrm{abcd}$ & 5.64 abcdef & 0.45 & 43.5 defgh \\
\hline 13 & ETBW 7268 & $15.5 \mathrm{abcd}$ & 3.1 & 9.30 abcde & 4.16 cdef & 0.44 & $46.0 \mathrm{abcd}$ \\
\hline 14 & ETBW 7174 & $13.0 \mathrm{~d}$ & 2.8 & 8.55 bcde & 3.04 ef & 0.35 & 44.0 cdefgh \\
\hline 15 & ETBW 7220 & $15.5 \mathrm{abcd}$ & 2.6 & $14.57 \mathrm{a}$ & $7.10 \mathrm{abc}$ & 0.49 & 43.0 defghi \\
\hline 16 & ETBW 7221 & $14.0 \mathrm{~cd}$ & 2.8 & $7.20 \mathrm{de}$ & 2.95 ef & 0.41 & $36.0 \mathrm{n}$ \\
\hline 17 & ETBW 7227 & $16.5 \mathrm{abcd}$ & 2.4 & $13.42 \mathrm{abc}$ & 5.87 abcde & 0.43 & 41.5 efghij \\
\hline 18 & ETBW 7239 & $13.5 \mathrm{~cd}$ & 3.1 & 11.78 abcde & 5.63 abcdef & 0.47 & $37.5 \mathrm{Imn}$ \\
\hline 19 & ETBW 7160 & $17.0 \mathrm{abcd}$ & 2.7 & 9.12 abcde & 3.87 def & 0.42 & 41.0 ghijk \\
\hline 20 & ETBW 7161 & $16.5 \mathrm{abcd}$ & 2.8 & $12.40 \mathrm{abcd}$ & 5.78 abcdef & 0.47 & 43.0 defghi \\
\hline 21 & ETBW 7191 & 16.0 abcd & 3.2 & 11.22 abcde & 5.22 abcdef & 0.47 & 44.5 bcdefg \\
\hline 22 & ETBW 7199 & $19.5 \mathrm{a}$ & 3.3 & $13.04 \mathrm{abc}$ & $6.21 \mathrm{abcd}$ & 0.47 & 42.5 defghi \\
\hline 23 & ETBW 7182 & $16.5 \mathrm{abcd}$ & 3.2 & $12.57 \mathrm{abcd}$ & $6.09 \mathrm{abcd}$ & 0.48 & 40.5 hijkl \\
\hline 24 & ETBW 7194 & $16.5 \mathrm{abcd}$ & 3.3 & 8.40 bcde & 3.68 def & 0.43 & 43.0 defghi \\
\hline 25 & ETBW 7204 & $13.5 \mathrm{~cd}$ & 3.3 & $12.79 \mathrm{abcd}$ & $6.22 \mathrm{abcd}$ & 0.48 & 43.5 defgh \\
\hline 26 & ETBW 7234 & $15.5 \mathrm{abcd}$ & 3.2 & 10.65 abcde & 4.77 abcdef & 0.44 & $37.0 \mathrm{mn}$ \\
\hline 27 & ETBW 7164 & $19.0 \mathrm{ab}$ & 2.8 & 9.77 abcde & 4.00 def & 0.41 & 43.0 defghi \\
\hline 28 & ETBW 7195 & $14.5 \mathrm{bcd}$ & 2.8 & 9.18 abcde & 3.82 def & 0.41 & 41.5 FGHIJ \\
\hline 29 & ETBW 7244 & $13.5 \mathrm{~cd}$ & 3.2 & 9.89 abcde & 3.69 def & 0.38 & 42.5 defghi \\
\hline 30 & ETBW 7258 & $16.0 \mathrm{abcd}$ & 3.2 & 11.89 abcde & 5.26 abcdef & 0.44 & 45.0 abcdef \\
\hline 31 & ETBW 7264 & $17.0 \mathrm{abcd}$ & 2.9 & $13.25 \mathrm{abc}$ & $6.31 \mathrm{abcd}$ & 0.47 & 43.5 defgh \\
\hline 32 & ETBW 7215 & $13.5 \mathrm{~cd}$ & 2.8 & $13.65 \mathrm{abc}$ & $6.26 \mathrm{abcd}$ & 0.45 & 41.5 fghij \\
\hline 33 & ETBW 7156 & $16.0 \mathrm{abcd}$ & 2.8 & 7.89 cde & 3.47 def & 0.44 & 41.0 ghijk \\
\hline 34 & ETBW 7247 & $16.50 \mathrm{abcd}$ & 3.3 & $12.37 \mathrm{abcd}$ & 5.01 abcdef & 0.40 & 42.0 efghi \\
\hline 35 & Danda'a & $13.5 \mathrm{~cd}$ & 2.9 & 9.10 abcde & 4.55 bcdef & 0.45 & $33.5 \circ$ \\
\hline \multirow[t]{3}{*}{36} & ETBW 7175 & $16.0 \mathrm{abcd}$ & 3.2 & 10.41 abcde & 5.04 abcdef & 0.48 & 40.5 hijkl \\
\hline & Mean & 15.92 & 3.02 & 11.19 & 5.08 & 0.45 & 41.99 \\
\hline & CV (\%) & 7.36 & 12.84 & 12.96 & 15.10 & 11.83 & 2.40 \\
\hline
\end{tabular}

\section{Principal Component Analysis}

The results of the principal component analysis for Gitilo and Guduru sites are presented in the Table 7 and 8 respectively. At Gitilo, the first four PCs accounted bout $81.06 \%$ of the total phenotypic variation in the genotypes. The PC1 accounted about $30.73 \%$ variations in the genotypes; and DH, GFP, SPL, SPS, BY, and GY are among the traits which contributed more to the phenotypic variations in the PC1; while $\mathrm{DH}, \mathrm{GFP}, \mathrm{PH}, \mathrm{SPL}$, and TSW showed more disparities to show differentiation in the genotypes in PC2 (21.62\%). At Guduru, the first three PCs (PC1, PC2, and PC3) contributed about $77.01 \%$ to the total phenotypic variations in the genotypes. All traits
(DH, PH, SPL, SPS, BY, GY and HI) had more contributions in the PC1 $(40.49 \%)$; while PH, SPL, GY and $\mathrm{HI}$ had more contributions to the phenotypic variations in PC2 (20.68\%).

\section{Correlation Analysis}

The results of phenotypic correlation analysis were presented in Table 9 and 10 for Gitilo and Guduru respectively. At Gitilo, GY had strong and positive associations with SPL and BY, indicating that longer spike genotypes had more kernels/yield than the short spike types. Genotypes with higher biomass due to high productive tillers had higher GY and productivity than 
Table 6: Mean values for different yield and other yield components in bread wheat genotypes tested at Guduru, 2013

\begin{tabular}{|c|c|c|c|c|c|c|c|}
\hline S/No. & Genotypes & SPS & NGPS & BY $\left(\mathrm{t} \mathrm{ha}^{-1}\right)$ & GY $\left(\mathrm{t} \mathrm{ha}^{-1}\right)$ & HI & TKW \\
\hline 1 & ETBW 7178 & $12.7 \mathrm{ab}$ & 2.6 & $5.05 \mathrm{abc}$ & $1.76 \mathrm{abcde}$ & $0.34 \mathrm{ab}$ & 37.5 \\
\hline 2 & ETBW 7252 & $12.5 \mathrm{ab}$ & 2.3 & $4.68 \mathrm{abc}$ & $1.22 \mathrm{abcde}$ & $0.26 \mathrm{~b}$ & 36.0 \\
\hline 3 & ETBW 7238 & $9.7 \mathrm{ab}$ & 3.0 & $5.31 \mathrm{abc}$ & $2.03 \mathrm{abcde}$ & $0.38 a b$ & 38.0 \\
\hline 4 & ETBW 7198 & $9.0 a b$ & 2.8 & $5.70 a b c$ & 1.29 abcde & $0.24 \mathrm{~b}$ & 34.0 \\
\hline 5 & KUBSA & $13.1 \mathrm{a}$ & 2.6 & $5.63 \mathrm{abc}$ & $1.71 \mathrm{abcde}$ & $0.30 \mathrm{ab}$ & 33.0 \\
\hline 6 & ETBW 7237 & $11.0 \mathrm{ab}$ & 3.1 & $4.59 \mathrm{abc}$ & 1.18 abcde & $0.25 \mathrm{~b}$ & 38.5 \\
\hline 7 & ETBW 7171 & $8.7 \mathrm{ab}$ & 2.3 & $3.14 \mathrm{c}$ & $1.10 \mathrm{cde}$ & $0.36 \mathrm{ab}$ & 36.0 \\
\hline 8 & ETBW 7208 & $11.1 \mathrm{ab}$ & 3.1 & $3.20 \mathrm{c}$ & $0.97 \mathrm{de}$ & $0.32 a b$ & 41.5 \\
\hline 9 & ETBW 7236 & $13.53 \mathrm{a}$ & 2.6 & $7.08 \mathrm{abc}$ & $2.67 \mathrm{abcde}$ & $0.37 \mathrm{ab}$ & 37.0 \\
\hline 10 & ETBW 7248 & $9.1 \mathrm{ab}$ & 2.3 & $6.46 \mathrm{abc}$ & $2.96 a b$ & $0.45 a b$ & 32.5 \\
\hline 11 & ETBW 7173 & $13.2 \mathrm{a}$ & 3.1 & $4.76 \mathrm{abc}$ & $3.01 \mathrm{a}$ & $0.64 \mathrm{a}$ & 31.5 \\
\hline 12 & ETBW 7235 & $11.5 \mathrm{ab}$ & 2.5 & $5.13 \mathrm{abc}$ & $1.91 \mathrm{abcde}$ & $0.38 a b$ & 35.5 \\
\hline 13 & ETBW 7268 & $12.7 \mathrm{ab}$ & 2.6 & $3.38 \mathrm{c}$ & $0.93 \mathrm{de}$ & $0.27 \mathrm{~b}$ & 38.5 \\
\hline 14 & ETBW 7174 & $9.8 \mathrm{ab}$ & 2.3 & $6.66 \mathrm{abc}$ & $1.81 \mathrm{abcde}$ & $0.26 \mathrm{~b}$ & 34.0 \\
\hline 15 & ETBW 7220 & $11.1 \mathrm{ab}$ & 2.6 & $4.90 \mathrm{abc}$ & 1.73 abcde & $0.36 \mathrm{ab}$ & 35.5 \\
\hline 16 & ETBW 7221 & $8.9 \mathrm{ab}$ & 2.45 & 4.25 bc & $1.05 \mathrm{cde}$ & $0.25 \mathrm{~b}$ & 33.0 \\
\hline 17 & ETBW 7227 & $10.7 \mathrm{ab}$ & 3.5 & $4.82 \mathrm{abc}$ & $1.55 \mathrm{abcde}$ & $0.34 \mathrm{ab}$ & 34.5 \\
\hline 18 & ETBW 7239 & $12.0 \mathrm{ab}$ & 2.3 & $3.10 \mathrm{c}$ & $1.11 \mathrm{cde}$ & $0.36 a b$ & 32.5 \\
\hline 19 & ETBW 7160 & $10.9 a b$ & 2.6 & $3.82 \mathrm{bc}$ & $0.91 \mathrm{de}$ & $0.28 \mathrm{~b}$ & 31.0 \\
\hline 20 & ETBW 7161 & $9.0 \mathrm{ab}$ & 2.1 & $4.55 \mathrm{abc}$ & $1.01 \mathrm{cde}$ & $0.22 \mathrm{~b}$ & 30.5 \\
\hline 21 & ETBW 7191 & $11.5 \mathrm{ab}$ & 2.9 & $6.20 \mathrm{abc}$ & $1.86 \mathrm{abcde}$ & $0.30 \mathrm{ab}$ & 30.5 \\
\hline 22 & ETBW 7199 & $11.3 \mathrm{ab}$ & 3.1 & $5.27 \mathrm{abc}$ & $2.36 \mathrm{abcde}$ & $0.45 a b$ & 34.0 \\
\hline 23 & ETBW 7182 & $11.8 \mathrm{ab}$ & 2.4 & $6.17 \mathrm{abc}$ & $2.79 \mathrm{abcd}$ & $0.45 a b$ & 38.0 \\
\hline 24 & ETBW 7194 & $9.2 \mathrm{ab}$ & 2.6 & $4.91 \mathrm{abc}$ & $1.57 \mathrm{abcde}$ & $0.33 a b$ & 33.0 \\
\hline 25 & ETBW 7204 & $11.0 \mathrm{ab}$ & 2.6 & $5.54 \mathrm{abc}$ & $1.87 \mathrm{abcde}$ & $0.33 a b$ & 41.0 \\
\hline 26 & ETBW 7234 & $11.8 \mathrm{ab}$ & 2.8 & $4.13 \mathrm{bc}$ & $1.79 \mathrm{abcde}$ & $0.43 a b$ & 37.0 \\
\hline 27 & ETBW 7164 & $7.6 \mathrm{~b}$ & 2.6 & $3.41 \mathrm{c}$ & $1.01 \mathrm{cde}$ & $0.29 \mathrm{ab}$ & 37.5 \\
\hline 28 & ETBW 7195 & $10.9 \mathrm{ab}$ & 2.3 & $3.59 \mathrm{c}$ & 0.78 e & $0.22 \mathrm{~b}$ & 38.5 \\
\hline 29 & ETBW 7244 & $9.0 \mathrm{ab}$ & 2.9 & 3.82 bc & $1.00 \mathrm{cde}$ & $0.26 \mathrm{~b}$ & 35.0 \\
\hline 30 & ETBW 7258 & $11.5 \mathrm{ab}$ & 3.2 & $6.05 a b c$ & $2.87 \mathrm{abc}$ & $0.47 a b$ & 31.5 \\
\hline 31 & ETBW 7264 & $11.3 \mathrm{ab}$ & 2.8 & $6.79 \mathrm{abc}$ & $2.32 \mathrm{abcde}$ & $0.36 \mathrm{ab}$ & 38.0 \\
\hline 32 & ETBW 7215 & $11.8 \mathrm{ab}$ & 2.4 & $8.74 \mathrm{a}$ & 1.72 abcde & $0.19 \mathrm{~b}$ & 31.5 \\
\hline 33 & ETBW 7156 & $9.3 \mathrm{ab}$ & 3.2 & $5.41 \mathrm{abc}$ & $1.34 \mathrm{abcde}$ & $0.25 \mathrm{~b}$ & 31.5 \\
\hline 34 & ETBW 7247 & $11.0 \mathrm{ab}$ & 2.7 & $8.07 \mathrm{ab}$ & $2.50 \mathrm{abcde}$ & $0.31 \mathrm{ab}$ & 41.0 \\
\hline 35 & Danda'a & $11.8 \mathrm{ab}$ & 2.4 & $5.83 \mathrm{abc}$ & 1.38 abcde & $0.23 \mathrm{~b}$ & 33.0 \\
\hline \multirow[t]{3}{*}{36} & ETBW 7175 & $8.5 \mathrm{ab}$ & 2.6 & $3.46 \mathrm{c}$ & $1.13 \mathrm{cde}$ & $0.32 a b$ & 35.5 \\
\hline & Mean & 10.81 & 2.69 & 5.10 & 1.67 & 0.33 & 35.19 \\
\hline & CV (\%) & 11.92 & 19.68 & 21.49 & 27.40 & 27.56 & 8.19 \\
\hline
\end{tabular}

those with low biomass yield and less productive tillers. Therefore, simultaneous selections for the above traits for individual genotype will improve both traits. $\mathrm{DH}$ was highly but negatively correlated with GFP, indicating those genotypes with longer DH had short GFP and vice versa. If the two traits are not controlled by similar or linked genes, selection could be done separately. SPL was positively and highly correlated with SPS indicating the two traits could simultaneously be improved through selection. $\mathrm{DH}$ and $\mathrm{DM}$ were positively and strongly associated; and $\mathrm{PH}$ and SPL were also positively and strongly associated; But GFP and SPS were highly but negatively associated. TSW had positive and strong association with $\mathrm{PH}$ and SPS, indicating longer stalks genotypes had higher TSW and productivity than the short stalk types; though longer stalks genotypes are not important due to the lodging effect in wheat.

At Guduru, GY was significantly and positively correlated with SPS, BY and $\mathrm{HI}$, hence higher biomass with more productive tillers give more grain yield than others. Similarly, higher SPS are highly associated with higher GY. Both traits are important yield components which could be used for selection criteria during genotypes evaluation for better adaptabilities to the environment. BY was also positively and significantly correlated with $\mathrm{DH}$ and SPL; and SPS was positively and highly correlated with $\mathrm{PH}$ and SPL.
Table 7: Principal component analysis for different agronomic traits of wheat genotypes tested at Gitilo, 2013

\begin{tabular}{ccccc}
\hline Traits & PC1 & PC2 & PC3 & PC4 \\
\hline DH & 0.374 & -0.494 & 0.190 & 0.129 \\
DM & 0.186 & -0.146 & -0.049 & 0.896 \\
GFP & -0.334 & 0.466 & -0.239 & 0.306 \\
PH & 0.069 & 0.517 & 0.241 & 0.116 \\
SPL & 0.389 & 0.321 & 0.125 & -0.114 \\
SPS & 0.369 & 0.102 & 0.445 & -0.134 \\
BY & 0.425 & 0.152 & -0.515 & -0.056 \\
GY & 0.460 & 0.113 & -0.474 & -0.078 \\
TKW & 0.170 & 0.316 & 0.380 & 0.187 \\
Eigen values & 2.766 & 1.950 & 1.560 & 1.026 \\
Proportion $(\%)$ & 30.73 & 21.62 & 17.30 & 11.40 \\
Cumulative variance(\%) & 30.73 & 52.36 & 69.66 & 81.06 \\
\hline
\end{tabular}

Table 8: Principal component analysis for different agronomic traits of wheat genotypes tested at Guduru, 2013

\begin{tabular}{cccc}
\hline Traits & Prin1 & Prin2 & Prin3 \\
\hline DH & 0.358 & 0.241 & -0.100 \\
PH & 0.298 & 0.432 & 0.496 \\
SPL & 0.363 & 0.335 & -0.260 \\
SPS & 0.423 & 0.162 & 0.417 \\
BY & 0.412 & -0.010 & -0.587 \\
GY & 0.472 & -0.479 & -0.122 \\
HI & 0.281 & -0.623 & 0.378 \\
Eigen values & 2.830 & 1.450 & 1.110 \\
Proportion (\%) & 40.49 & 20.68 & 15.84 \\
Cumulative variance (\%) & 40.49 & 61.17 & 77.01 \\
\hline
\end{tabular}


Table 9: Pearson correlation coefficients for agronomic traits of wheat genotypes tested at Gitilo, 2013

\begin{tabular}{ccllllllll}
\hline Traits & DH & DM & GFP & PH & SPL & SPS & BY & GY & TKW \\
\hline DH & 1.00 & $0.41^{*}$ & $-0.85^{* *}$ & -0.24 & 0.09 & 0.31 & 0.15 & 0.21 & 0.02 \\
DM & & 1.00 & 0.01 & -0.03 & 0.07 & 0.06 & 0.14 & 0.15 & 0.04 \\
GFP & & & 1.00 & 0.24 & -0.13 & $-0.35^{*}$ & -0.09 & -0.18 & 0.03 \\
PH & & & & 1.00 & $0.37^{*}$ & 0.20 & 0.05 & 0.00 & $0.34^{*}$ \\
SPL & & & & & 1.00 & $0.52^{* *}$ & $0.36^{*}$ & $0.41^{*}$ & 0.23 \\
SPS & & & & & & 1.00 & 0.11 & 0.18 & $0.36^{*}$ \\
BY & & & & & & & 1.00 & $0.96^{* *}$ & 0.05 \\
GY & & & & & & & & 1.00 & 0.06 \\
TKW & & & & & & & & & 1.00 \\
\hline
\end{tabular}

Table 10: Pearson correlation coefficients for agronomic traits of wheat genotypes tested at Gudru, 2013

\begin{tabular}{cccccccc}
\hline Traits & DH & PH & SPL & SPS & BY & GY & HI \\
\hline DH & 1.00 & 0.32 & 0.31 & 0.33 & $0.37^{*}$ & 0.29 & 0.07 \\
PH & & 1.00 & 0.30 & $0.53^{* *}$ & 0.11 & 0.08 & 0.05 \\
SPL & & & 1.00 & $0.38^{*}$ & $0.45^{\star *}$ & 0.27 & -0.03 \\
SPS & & & & 1.00 & 0.26 & $0.37^{*}$ & 0.29 \\
BY & & & & & 1.00 & $0.66^{* *}$ & 0.02 \\
GY & & & & & & 1.00 & $0.74^{\star *}$ \\
HI & & & & & & & 1.00 \\
\hline
\end{tabular}

\section{DISCUSSION}

At Gitilo (highland) and Guduru (midland) analyses of variance showed that significant differences among the genotypes for most of the traits indicating the genotypes are variable and can be used as source materials for subsequent breeding works. The range values were higher for the traits including DF, DM, GFP, PH and TSW; while coefficients of variations for traits were higher at Guduru (midland) than at Gitilo (highland) showing the more prominent effect of environment at Guduru than at Gitilo. Because at Guduru the rainfall amount was shorter and lower in intensity; and temperature was high as compared to the highland with longer rainfall period and low temperature. In addition, the soil characteristics of the two sites are different (Deressa, 2013). The mean values for all traits for the genotypes at Gitilo are higher than at Guduru except for tiller number per plant indicating the highland area is suitable for wheat production. The number of tiller per plant at Guduru is higher than at Gitilo. The mean values for productivity which could be expressed as seed yield per hectare, biological yield per hectare and harvest index are higher and it was 2, 3 and 1.4 times respectively higher than at Guduru. Wheat is suitably grown at higher altitude or cooler environments than warmer areas (Geleta, 2015) and in Ethiopia also wheat is suitably grown in range of altitudes between 2200 m.a.s.I and 2800 m.a.s.I (Geleta and Grausgruber, 2011). In present study Gitilo represents higher altitude areas while Guduru represents low altitude areas. Therefore, in Ethiopia altitude affects the rainfall distribution and temperature which is directly connected to photosynthetic efficiency of the crop.

The performances of the genotypes with respect to biological yield, grain yield, harvest index and thousand seed weight are different in the two sites. For instances, the rank of the genotypes with respect to the performance of the indicated traits are different. Regarding grain yield, the top ten best performed varieties at Gitilo include ETBW 7238, ETBW 7198, ETBW 7220, ETBW 7208, ETBW 7252, ETBW 7264, ETBW 7215, ETBW 7204, ETBW 7199, and ETBW 7182. The highest yielder variety ETBW 7238 gave $7.56 \mathrm{t} \mathrm{ha}^{-1} ; 14.11 \mathrm{t} \mathrm{ha}^{-1} ; 0.54$ and $45.5 \mathrm{~g}$ for grain yield, biological yield, harvest index and thousand seed weight respectively. However; at Guduru the top ten ranking varieties for grain yield include ETBW 7173, ETBW 7248, ETBW 7258, ETBW 7182, ETBW 7236, ETBW 7247, ETBW 7199, ETBW 7264, ETBW
7238, ETBW 7235. The highest yielder variety ETBW 7173 gave $3.01 \mathrm{t} \mathrm{ha}^{-1}, 4.76 \mathrm{t} \mathrm{ha}^{-1}, 0.64$ and $31.5 \mathrm{~g}$ for grain yield, biological yield, harvest index and thousand seed weight, respectively. Therefore, the ranks of the genotypes were changed due to genotype by environment interaction which could force the breeders to carry out adaptation trial in different environments. Effect of environment on quantitative traits is high since quantitative traits are controlled by many genes with small individual effects (Allard, 1960). In agreement with present findings, Girma et al. (2001) reported that moisture stress environments are the limiting factors in bread wheat production and expansion in Ethiopia and there is high interaction of genotypes with environment.

The results of the principal component analysis for traits showed that the first four and three PCs at Gitilo and Guduru, respectively, accounted about $81.06 \%$ and 77.01 $\%$ of the total phenotypic variations in the genotypes indicating that variations among the genotypes are due to majorly the traits considered. However, there were unexplained variables accounted for variations which could be attributed to environment. Phenotypic traits including DH, GFP, SPL, SPS, BY, and GY; and DH, PH, SPL, SPS, BY, GY and $\mathrm{HI}$ are among the traits which contributed more to the phenotypic variations in the PC1 at Gitilo and Guduru respectively.

At Gitilo, GY had strong and positive associations with SPL and BY, indicating that they are direct and indirect contributors to grain yield and could be considered for indirect selection for grain yield. $\mathrm{DH}$ was highly but negatively correlated with GFP, indicating those genotypes with longer in $\mathrm{DH}$ had short GFP and vice versa. Corroborating the present findings, Guendouz and Maamari (2012) reported the negative correlation of days to heading and velocity of grain filling in durum wheat varieties. These could be due to two reasons: 1 . The two traits might be controlled by linked genes or pleotropic genes, and selection could not be easy to improve both traits as the same time; 2. Genotypes with higher $\mathrm{DH}$ and Shorter GFP are due to external environmental factors such as late types genotypes encounter terminal moisture stress and higher temperature that hastens maturity or grain filling. However, further evidence could support the two assumptions. Spike length was positively and significantly correlated with SPS indicating the two traits could simultaneously be improved through selection. Yahaya (2014) also reported positive significant 
Negash Gelata et alo,

correlation of yield with length of spike, spike lets per spike, weight of main spike and plant height. DH and DM were positively and strongly associated; and $\mathrm{PH}$ and SPL were also positively and strongly associated; but GFP and SPS were significantly but negatively associated. TSW had positive and strong association with $\mathrm{PH}$ and SPS, indicating longer stalk genotypes had higher TSW and more productive than the short stalk types; though longer stalks genotypes are not important due to the lodging effect in wheat. At Guduru, GY was significantly and positively correlated with SPS, BY and $\mathrm{HI}$, hence higher biomass with more productive tillers give more grain yield than others. Belay et al. (1993) reported strong associations of grain yield with thousand kernel weight and tiller number per plant in tetraploid wheat from Ethiopia. Fischer and Kertesz (1976) concluded from their experiment that harvest index is the best predictor of grain yield in wheat. Similarly, higher SPS are significantly associated with higher GY. Both traits are important yield components which could be used for indirect selection criteria during genotypes evaluation for better adaptabilities to the specific environment. BY was also positively and significantly correlated with $\mathrm{DH}$ and SPL; and SPS was positively and highly correlated with $\mathrm{PH}$ and SPL. Girma et al. (2001) also found strong associations of grain yield with test weight, plant height and thousand kernel weight in moisture stress environments for bread wheat varieties in Ethiopia.

\section{CONCLUSIONS}

The results of ANOVA showed that significant differences among the breeding lines at both locations for $\mathrm{DH}, \mathrm{PH}, \mathrm{SPL}$, SPS, BY, GY and TKW; while for DM and GFP at highland (Gitilo) and for $\mathrm{HI}$ at midland (Guduru). High coefficients of variations were observed at Guduru as compared to at Gitilo indicating that high influence of environment on the performances of the genotypes at lowland areas. Higher mean values were observed for all traits at Gitilo than at Guduru indicating the high potential of the highland areas as compared to the lowland areas for wheat production. The rank of genotypes for grain yield potential were changed with respect to the two sites indicating genotype by environment interaction on the yield and yield related traits of wheat. However, at both locations, genotypes which showed superior performances compared to the standard checks 'Danada' and 'Kubsa' for biomass yield, grain yield, thousand kernel weight and harvest index include: ETBW7238, ETBW7235, ETBW7220, ETBW7191, ETBW7199, ETBW7182, ETBW7204, ETBW7258, ETBW7264, ETBW7247; and these genotypes are selected for multilocation trials for wide adaptations. Principal components analysis showed that the first four PCs contributed about $81.06 \%$ of the total phenotypic variation in the genotypes at Gitilo; while the first three PCs contributed about 77.01 $\%$ to the total phenotypic variations in the genotypes at Guduru site. Analysis for traits association showed that at Gitilo, GY had strong and positive associations with SPL and $B Y$, indicating that genotypes with long spike had more kernels or yield than the short spike types. Similarly, at Guduru, GY was significantly and positively correlated with SPS, BY and HI. At both locations, traits which showed strong associations with GY could be used for indirect selection criteria during genotypes evaluation for better adaptabilities to the environment. In conclusion separate breeding strategies are needed for highland and lowland areas for wheat production and the need to evaluate genotypes for their adaptabilities in the two environments.
Sci. Technol. Arts Res. J., Jan-March 2015, 4(1): 01-08

\section{Conflict of Interest}

Conflict of interest none declared

\section{Acknowledgments}

The authors thank Kulumsa Agricultural Research Centre for providing the planting materials. The authors also thank Wollega University for financing the project.

\section{REFERANCE}

Allard, R.W. (1960). Principles of Plant Breeding. John Wiley and Sons. Inc. New York.

Belay, G., Tesemma, T., Becker, H.C. and Merker, A. (1993). Variation and interrelationships of agronomic traits in Ethiopian tetraploid wheat landraces. Euphytica 71(3): 181-188.

CSA (2014). Agricultural Sample Survey in 2013/2014 (2006 E.C.). Report on Area and Production of major crops for Private Peasant Holdings, Meher Season. Vol. 1, Statistical Bulletin Number 532, Addis Ababa, Ethiopia.

Deressa, A. (2013). Evaluation of soil acidity in agricultural soils of smallholder farmers in south western Ethiopia. Science, Technology and Arts Research Journal 2(2):1-6.

Dewey, D.R., Lu, K.H. (1959). A correlation and path coefficient analysis of components of crested wheat grass seed production. Agronomy Journal 51: 515-518.

Fischer, R. A., Kertesz, Z. (1976). Harvest index in spaced populations and grain weight in microplots as indicators of yielding ability in spring wheat. Crop Science 16(1):55-59.

Geleta, N. (2015). Patterns of variation for phenotypic traits in Tetraploid Wheat (Triticum turgidum L.) populations of Ethiopia. Agricultural and Biological Sciences Journal 1(2): 42-51.

Geleta, N., Grausgruber, H. (2013a). Morphological and quality traits variation in tetraploid and hexaploid wheat accessions from Ethiopia. Agricultural Science Research Journal 3(8): 229-236.

Geleta, N., Grausgruber, H. (2013b). On-farm diversity and genetic erosion in Tetraploid wheat landraces in Ambo and Dandi Districts, West Shewa, Ethiopia. Science, Technology and Arts Research Journal 2(1): 1-9.

Geleta, N., Grausgruber, H. (2011). Phenotypic variation of Ethiopian hexaploid wheat accessions. East African Journal of Sciences 5(2): 89-97.

Gomez, K.A., Gomez, A.A. (1984). Statistical Procedures for Agricultural Research, $2^{\text {nd }}$ ed. John Wiley \& Sons, USA.

Girma, B., Alemayehu, Z., Gelalcha, S. (2001). Drought tolerance of some bread wheat genotypes in Ethiopia. African Crop Science Journal 9(2): 385-392.

Guendouz, A., Maamari, K. (2012). Grain-filling, chlorophyll content in relation with grain yield component of durum wheat in a mediterranean environment African Crop Science Journal 20(1):31-37.

Lelley, J. (1976). Wheat breeding: Theory and practice. Akademial Kiado, Budapest.

Ribaut, J.M., William, H.M., Khairallah, M., Worland, A.J., Hoisington, D. (2001). Genetic basis of physiological traits, pp. 29-47. In: Reynolds, M.P., Ortiz- Monasterio, J.I., McNab, A. (Ed.) Application of Physiology in Wheat Breeding. Mexico, D.F. CIMMYT.

Yahaya, Y. (2014). Estimate of genetic variability and correlation coefficients for some quantitative characters in bread wheat (Triticum aestivum L.). World Journal of Agricultural Sciences 2(7):163-167.

Zaharieva, M., Geleta, N., Hakimi, A.A., Misra, S.C. (2010). Cultivated emmer wheat (Triticum dicoccon Schrank) an old crop with promising future: a review. Genetic Resources and Crop Evolution 57: 937-962. 